\title{
Resíduos dos serviços de saúde: a responsabilidade ambiental na visão dos profissionais da Atenção Primária
}

\author{
Health services residues: Environmental responsibility in Primary Care Professional view \\ Residuos de servicios sanitarios: responsabilidad ambiental en la visión de los profesionales de
} Atención Primaria

Recebido: 22/02/2021 | Revisado: 28/02/2021 | Aceito: 08/03/2021 | Publicado: 16/03/2021

Luzibênia Leal de Oliveira

ORCID: https://orcid.org/0000-0002-8549-370X Universidade Federal de Campina Grande, Brasil

E-mail: luzibenia@gmail.com

Patrício Marques de Souza

ORCID: https://orcid.org/0000-0002-2064-3818 Universidade Federal de Campina Grande, Brasil

E-mail: patriciomsouza@gmail.com

Francisco de Sales Clementino ORCID: https://orcid.org/0000-0001-8470-4694 Universidade Federal de Campina Grande, Brasil

E-mail: fclementino67@yahoo.com.br

Jank Landy Simôa Almeida

ORCID: https://orcid.org/0000-0001-8466-4880 Universidade Federal de Campina Grande, Brasil

E-mail: jankalmeida@gmail.com

Nívea Vilar Cardoso

ORCID: https://orcid.org/0000-0001-6025-1678 Universidade Federal de Campina Grande, Brasil E-mail: niveavilar@gmail.com

Lara Maria Alves de Carvalho ORCID: https://orcid.org/0000-0001-7476-0051 Universidade Federal de Campina Grande, Brasil E-mail: 1a3107228@gmail.com

\begin{abstract}
Resumo
Objetivou-se analisar o conhecimento dos profissionais da Atenção Primária em Saúde acerca da responsabilidade da produção de resíduos de serviços de saúde até a disponibilização para o meio ambiente. Trata-se de uma estudo exploratório-descritivo e transversal, que utilizou a abordagem qualitativa, realizada em 36 Unidades Básicas de Saúde presentes nos nove Distritos Sanitários do município de Campina Grande, Paraíba. A amostra do estudo foi composta por 7 médicos, 24 enfermeiros, 04 dentistas, 16 auxiliares de enfermagem, 04 auxiliares de saúde bucal, totalizando 55 profissionais entrevistados. Os dados foram coletados de agosto de 2011 a janeiro de 2012 e analisados à luz dos postulados de Bardin (2016). Os resultados revelaram que os profissionais fazem claramente uma associação entre o gerenciamento inadequado dos resíduos com a contaminação ambiental e o surgimento de doenças. Outra questão levantada foi a falta de esclarecimento sobre gerenciamento de resíduos; condições inadequadas de trabalho; falta de apoio aos profissionais vítimas de acidentes com os resíduos. Os entrevistados ainda apontaram a coleta seletiva como uma alternativa para melhorar o gerenciamento dos resíduos. Com isso, pode-se concluir que o estudo pode ser de grande valia para gestores que desejem realizar mudanças na coleta de resíduos de serviços de saúde.
\end{abstract}

Palavras-chave: Resíduos; Atenção primária em saúde; Meio ambiente; Sociedade.

\begin{abstract}
This study aimed to analyze the professionals knowledge of Primary Health about the responsibility of the health services waste production until the release to the environment. This is an exploratory-descriptive and cross- sectional study using a qualitative approach, carried out in 36 Basic Health Units present in nine health districts of the Campina Grande city in the state of Paraiba. The study sample was composed by seven doctors, 24 nurses, 04 dentists, 16 nurse assistants, 04 oral health auxiliaries, totaling 55 professionals interviewed. Data were collected from august 2011 to january 2012 and analyzed in the light of the Bardin (2016) postulates. The results showed clearly that professionals make an association between inadequate waste management to environmental contamination and the onset of diseases. Other question referred was the lack of information on waste management; inadequate work conditions; lack of support for professionals victims of accidents with waste. The interviewees also indicated the selective waste collection as an
\end{abstract}


alternative to improve the management of residues. Thus, it can be concluded that the study can be of great value to managers who wish to make changes in the collection of waste from health services.

Keywords: Waste; Primary health care; Environment; Society.

\section{Resumen}

El objetivo fue analizar el conocimiento de los profesionales de Atención Primaria de Salud sobre la responsabilidad de producir residuos de los servicios de salud hasta su puesta a disposición del medio ambiente. Se trata de un estudio exploratorio-descriptivo y transversal, que utilizó el enfoque cualitativo, realizado en 36 Unidades Básicas de Salud presentes en los nueve Distritos Sanitarios del municipio de Campina Grande, Paraíba. La muestra del estudio estuvo conformada por 7 médicos, 24 enfermeras, 04 dentistas, 16 auxiliares de enfermería, 04 auxiliares de salud bucal, totalizando 55 profesionales entrevistados. Los datos se recopilaron desde agosto de 2011 hasta enero de 2012 y se analizaron a la luz de los postulados de Bardin (2016). Los resultados revelaron que los profesionales claramente establecen una asociación entre la gestión inadecuada de residuos y la contaminación ambiental y la aparición de enfermedades. Otra cuestión planteada fue la falta de aclaración sobre la gestión de residuos; condiciones de trabajo inadecuadas; Falta de apoyo a los profesionales víctimas de accidentes con residuos. Los encuestados también señalaron la recogida selectiva como una alternativa para mejorar la gestión de residuos. Así, se puede concluir que el estudio puede ser de gran valor para los gestores que deseen realizar cambios en la recogida de residuos de los servicios sanitarios.

Palabras clave: Residuos; Atención primaria de salud; Medio ambiente; Sociedad.

\section{Introdução}

O gerenciamento adequado dos resíduos gerados pelos estabelecimentos de saúde é fundamental para a promoção da saúde, qualidade de vida e preservação do meio ambiente. Para tal, se faz necessário que os gestores: municipais, estaduais e federais, busquem conhecer profundamente o assunto e coloquem em prática a legislação e normatizações vigentes.

Como Oliveira e Júnior (2016) citam, para um correto planejamento de ações da gestão de resíduos sólidos em uma localidade, é necessário um período de estudos prospectivos. Por isso, panoramas e resoluções são continuamente criados para um melhor avanço da coordenação dos resíduos urbanos. A partir disso, as atividades que envolvem etapas do gerenciamento de Resíduos de Serviços de Saúde (RSS), sejam eles privados ou públicos, civis, filantrópicos ou militares, incluindo, também, aqueles que exercem ações de ensino e pesquisa, foram regulamentadas pela Resolução da Diretoria Colegiada - RDC 222/18 da Agência Nacional de Vigilância Sanitária (2018). Nela, foi definido que todos os serviços, nos quais as atividades estejam correlacionadas com a atenção à saúde humana ou animal devem observar os termos e condições específicas para seu licenciamento ambiental. Essa resolução, porém, não se aplica às fontes radioativas seladas, que precisam seguir as normas e determinações da Comissão Nacional de Energia Nuclear (CNEN), assim como as indústrias de produtos sob vigilância sanitária (ANVISA, 2018).

Partindo do princípio de que quanto maior a população maior é a geração dos RSU, a Associação Brasileira de Empresas de Limpeza Pública e Resíduos Especiais - ABRELPE - (2020) afirma que no Brasil, são geradas, anualmente, quase 79 milhões de toneladas de resíduos sólidos urbanos. Comparando os anos de 2010 com 2019 houve um aumento de 18,5\%, com uma diferença de mais de 12 milhões de toneladas de resíduos. Em relação à participação das regiões do país no total de RSU coletados, a Sudeste é responsável por 49,8\%; a Nordeste por 24,9\%; a Sul por 10,4\%; a Norte por 7,4\% e a Centro-Oeste por 7,3\%. Ainda segundo a mesma Associação, apenas 59,5\% do lixo urbano coletado no Brasil recebeu a destinação correta: os aterros sanitários. O restante (equivalente a 29,4 milhões de toneladas) foi lançado em locais inadequados. Parte desse lixo urbano é constituído pelos resíduos de serviços de saúde (RSS), que representam risco à saúde e ao meio ambiente, se dispostos de forma incorreta.

Nessa perspectiva, quanto maior a população, maior é também o número de pessoas que manuseiam esses resíduos, colocando sua vida em risco, sejam elas dentro ou fora dos estabelecimentos geradores. Há, também, os riscos que podem afetar e agravar a comunidade hospitalar, principalmente nos grupos constituídos por pacientes em tratamento específicos e minuciosos, com grande comprometimento de seu sistema de defesa. O manejo inadequado dos resíduos sólidos da saúde pode causar, além 
do risco ambiental, perda da qualidade de vida das pessoas, mesmo com contato direto ou não com o material descartado (Cafure \& Patriarcha-Graciolli, 2015).

Diante dessas considerações, elaboramos a seguinte questão como norteadora do nosso estudo: que conduta os profissionais da Estratégia Saúde da Família vêm assumindo frente à problemática dos resíduos dos serviços de saúde? Destarte, diante a ideia descrita nessa perspectiva, elaborou-se o seguinte objetivo: analisar o conhecimento dos profissionais da Atenção Primária em Saúde acerca da responsabilidade da produção de RSS até a disponibilização para o meio ambiente.

Considerando a necessidade de ampliar a temática dos resíduos sólidos de serviços de saúde, acredita-se que o presente estudo possa estimular o processo de aprendizagem do gestor, gerente e demais trabalhadores dessas unidades, sensibilizandoos para a necessidade de uma melhor compreensão sobre o gerenciamento dos resíduos em Unidades de Saúde da Família.

\section{Metodologia}

Trata-se de uma pesquisa do tipo exploratório, descritivo e transversal, que utilizou a abordagem qualitativa, que, segundo Pereira, Shitsuka, Parreira e Shitsuka (2018), é importante a interpretação e opinião do pesquisador em questão. Realizada em 36 Unidades Básicas de Saúde presentes nos nove Distritos Sanitários (DSs), a pesquisa foi realizada no município de Campina Grande, Paraíba. A amostra do estudo foi composta por 7 médicos, 24 enfermeiros, 04 dentistas, 16 auxiliares de enfermagem, 04 auxiliares de saúde bucal, totalizando 55 profissionais entrevistados. Todos escolhidos de forma não probabilística e intencional, com a finalidade de englobar todos os distritos sanitários, além de equipes da zona urbana e rural. Para os critérios de definição dos sujeitos foram considerados a experiência mínima de um ano desses profissionais nesses serviços e geradores diretos ou em potencial de resíduos na área da saúde, acrescido do aceite para participar da pesquisa. Os dados foram coletados de agosto de 2011 a janeiro de 2012, por meio de entrevista individual, utilizando-se um roteiro semiestruturado contendo questões sobre formação profissional, tempo de trabalho e capacitação direcionada a ESF, além de perguntas relacionadas aos conceitos e práticas de Promoção da Saúde. Ressalta-se que todas as etapas foram realizadas após aprovação do Comitê de Ética em Pesquisa com Seres Humanos da Universidade Estadual da Paraíba (UEPB), (CAAE No 0334.0.133.000-11).

As entrevistas foram gravadas, com a anuência do entrevistado, para resguardar a integridade dos discursos. Os dados coletados foram codificados por letras e números referentes à formação profissional, com vistas a facilitar a apresentação no texto e garantir o anonimato dos entrevistados. As entrevistas tiveram seu conteúdo submetido à análise temática de conteúdo proposta por Bardin (2016), seguindo as seguintes etapas: pré-análise, exploração do material, tratamento dos dados e interpretação. A análise dos dados, organizados em unidades de significado, levou a identificação de categorias, que permitem a compreensão do fenômeno em estudo, por meio da descrição das vivências destes profissionais. Os nomes dos participantes foram ocultados e substituídos pela letra (E) seguida do número da entrevista para a apresentação de suas falas nos resultados.

\section{Resultados e Discussão}

Após a leitura dos depoimentos, elegemos as categorias temáticas, mediante as leituras dos depoimentos, associando aos objetivos preestabelecidos, buscando fundamentação na base teórica adotada.

\subsection{Primeira categoria: Resíduos Serviços de Saúde: efeitos indesejáveis e muitas vezes irreversíveis do ponto de vista sanitário e ambiental}

Os profissionais entrevistados fizeram claramente uma associação entre o gerenciamento inadequado dos resíduos com a contaminação do meio ambiente e o surgimento de doenças infecto-contagiosas e ocupacionais, conforme as falas abaixo: 
“A importância pra mim é que tem a ver com a nossa vida. Cuidar do meio ambiente significa cuidar da nossa saúde e quando a gente está produzindo lixo de forma assustadora isso vai repercutir nas gerações futuras, certo? Será que esse lixo não está contaminando o lençol freático? Será que esse lixo não 'tá sendo usado por pessoas que vivem do lixo e de forma equivocada?" (E 01).

“A necessidade seria a prevenção de doenças que são veiculadas nesse lixo, então a gente tem que ter cuidado e há necessidade que todos conheçam esse processo para não se contaminarem e a propagação dessas doenças não continuar acontecendo" (E 05).

O lixo hospitalar, quando manejado de forma correta, causa bem menos riscos. Esse manejo satisfatório, então, precisa ser realizado de forma completa, começando da geração deste lixo e indo até a sua destinação final (Fröhlich, 2016). Com o manejo inadequado deste resíduo sólido, é possível que o mesmo sirva de abrigo para vetores de doenças ou até provoque acidentes (Brasil, 2017).

Os RSS, ao serem disponibilizados de qualquer maneira em depósitos a céu aberto, ou em cursos de água, tornam possível a contaminação de mananciais de água potável, sejam superficiais ou subterrâneos. Com isso, podem propiciar a disseminação de doenças por meio de vetores que se multiplicam nestes locais ou que fazem dos resíduos, fonte de alimentação (Barbosa \& Barbosa, 2014). Entretanto, vale frisar que nem todo resíduo com origem em ambientes hospitalares é considerado infectante, uma vez que 70 a 90\% desses resíduos dos serviços de saúde deveriam ser resíduos comuns (Masieiro, 2020).

Baseado nas evidências descritas acima, faz-se necessário elaborar um plano de gerenciamento de resíduos de saúde (PGRSS), considerando as características e classificação dos resíduos gerados na instituição de saúde, a fim de que intervenções reais possam minimizar os riscos inerentes ao ambiente e às atividades desenvolvidas pelos profissionais. Além disso, sugere-se a implantação de atividades direcionadas ao treinamento e conscientização dos profissionais sobre os danos à saúde humana e ao meio ambiente.

Encontramos, também, nas falas dos participantes do estudo, referência aos agravos oriundos da utilização do mercúrio presente no amálgama odontológico. Isso é demonstrado na fala a seguir:

“[...] tudo que a gente lava nessa pia, cai atrás no terreno do posto, não tem uma coleta de esgoto aqui, aí querendo ou não vai cair alguma coisinha de material de mercúrio, porque às vezes solta umas bolinhas de mercúrio do amalgamador e eu acho que é perigoso, porque fica tudo aí no solo contaminando e isso pode trazer transtorno ambiental [...]" (E 09).

O mercúrio é um metal tóxico para os seres vivos e para o meio ambiente, sendo a exposição ocupacional uma das principais fontes de contaminação por esse metal. A forma metálica ou elementar é a mais prejudicial à saúde dos trabalhadores, além de seu predicado de bioacumulação, ou seja, a capacidade de se acumular nos tecidos biológicos (Anusavice, 2013; Homrich, Fernandes \& Vieira, 2014).

\subsection{Segunda categoria: A importância da formação de um espaço que propicie a problematização e conscientização sobre a responsabilidade dos $\mathrm{RSS}$}

Esse fato foi apontado nos discursos dos entrevistados, os quais apontam a falta de esclarecimento sobre gerenciamento dos resíduos sólidos, condições inadequadas de trabalho, responsabilidade na produção dos RSS, constituindo-se, no cotidiano, na transferência de riscos e na possibilidade de acidentes para os trabalhadores da saúde, sociedade e para o meio ambiente. 
"Eu acho que a grande maioria dos profissionais nem desperta, antes dessa pesquisa, nem a gente despertava para isso, que destino eи doи ао теи lixo... Eu acho que ninguém desperta, produzir a gente produz, mas despertar para a repercussão disso na natureza, a gente não desperta" (E 01).

"[...] a gente nunca teve treinamento relacionado a essa questão do gerenciamento dos resíduos sólidos, seria muito bom que todos os profissionais, inclusive os auxiliares de serviços gerais fossem capacitados, até mesmo saber como é dado o destino a esse lixo [...]" (E 11).

No espaço avaliado, ficou clara a afirmação dos participantes do estudo quanto à necessidade de uma educação contínua e permanente para o cuidado com os RSS. Esta estratégia poderá permitir a elaboração de um novo planejamento, voltado para as necessidades identificadas, com o objetivo de capacitar e, consequentemente, minimizar a exposição e a vulnerabilidade deles, aos acidentes com materiais perfurocortantes envolvendo sangue, fluidos corporais, dentre outros (Santos Junior, Batista, Almeida \& Abreu, 2014).

As medidas adequadas de manejo dos RSS contribuem em muito para a preservação da saúde ambiental e humana, uma vez que a segregação dos RSS no momento e local de sua geração (na fonte) permite reduzir o volume de resíduos perigosos e a incidência de acidentes ocupacionais dentre outros benefícios à saúde pública e ao meio ambiente.

Logo, além dos profissionais, se faz importante que tal conhecimento possa ser repassado desde o ensino básico, uma vez que essa institucionalização do saber pode gerar uma propagação social, estabelecendo um vínculo maior com a comunidade externa, pois os maiores propagadores, também, podem ser seus alunos e seus familiares, vindo a difundir a cultura que estão absorvendo da Rede de Ensino (Capponi, Ahlert, Dal'Asta \& Fiirst 2021).

\subsection{Terceira categoria: Ausência de Divulgação sobre as Normas, Regulamentos e Legislações Específicas de} Gerenciamento de RSS

Percebe-se nas falas abaixo uma carência de divulgação de normas, regulamentos e legislações específicas de gerenciamento de RSS.

“[...] é uma coisa que você ver que a Secretaria de Saúde, o gestor, não dá essa base pra gente, pra ninguém, eu já perguntei na Secretaria de Saúde se alguém sabe do PGRSS e eles não sabiam nem o que era isso, então fica difícil pra gente participar desse gerenciamento, onde realmente todos tem que participar, sem ter uma base legal, um fluxograma de todo esse lixo. Então fica difícil, a gente orientar as meninas da limpeza [...]” (E 03).

Com base no depoimento acima, fica claro que um pacto coletivo em busca da sustentabilidade é fundamental. Para isso, é necessário educação e conscientização de todos os trabalhadores e participantes da prestação de serviço (Borlina Tanaue, Mendes Bezerra, Cavalheiro \& Pisano, 2015).

Percebe-se também uma desarticulação da gestão municipal com os profissionais da Saúde da Família, pouco investimentos em capacitação, ausência de divulgação dos planos e projetos que orientam a Política de Gerenciamento dos RSS, dentre outros. Essa negligência faz contraponto à ideia da própria Constituição Federal (1988), que cita a importância das ações e serviços de saúde integralizados para uma melhor e mais completa assistência em saúde. 


\subsection{Quarta categoria: Coleta Seletiva: uma correta segregação dos resíduos no estabelecimento gerador.}

A possibilidade de estabelecer um programa de coleta seletiva foi algo sinalizado pelos profissionais conforme vemos nas falas abaixo, porém ficou notória a necessidade de apoio da gestão, como também a sensibilização contínua da população.

“[...] nós temos os lixeiros de coleta seletiva aí na entrada da unidade, que é de um projeto da universidade, é feito sala de espera sobre o assunto, mas a população ainda não está utilizando corretamente. É uma questão de educação da população, o que não é fácil, é uma questão de sensibilização mesmo [...]” (E 11).

Programas de minimização e reciclagem podem ser implantados em qualquer setor da sociedade, inclusive na área da saúde. Porém a implantação deste programa em serviços de saúde exige um minucioso diagnóstico, treinamento de funcionário, alterações de rotina, além de uma mudança de mentalidade e de hábitos (Ramos, 2013; Fröhlich, 2016).

\section{Considerações Finais}

Estudos como este são capazes de despertar nos gestores - secretário de saúde, coordenador de atenção básica, gerentes de distrito sanitários - o interesse em conhecer melhor todo o processo de gerenciamento dos resíduos na atualidade, identificar eventuais falhas e realizar as correções necessárias para que haja uma redução e destinação final dos resíduos gerados na atenção básica no município de Campina Grande - PB, servindo de exemplo para outros municípios brasileiros.

Para a realização de futuras pesquisas no tema, sugerimos a realização de investigações para aplicações das políticas públicas relacionadas ao saneamento básico, especialmente no tocante à destinação dos resíduos.

\section{Referências}

Agência Nacional de Vigilância Sanitária - ANVISA. (2018). Resolução RDC N ${ }^{\circ} 222$, de 28 de março de 2018 comentada. https://www20.anvisa.gov.br/segurancadopaciente/index.php/legislacao/item/resolucao-rdc-n-222-de-28-de-marco-de-2018-comentada.

Associação Brasileira de Empresas de Limpeza Pública e Resíduos Essenciais - ABRELPE. (2020). Panorama de Resíduos Sólidos no Brasil. https://abrelpe.org.br/panorama/.

Anusavice, K. J., Shen, C. \& Rawls, H.R. (2013). Phillips Materiais Dentários (12a ed.). Elsevier.

Barbosa, K. G. N., \& Barbosa, A. C. N. (2014). O Impacto do lixo na saúde e a problemática da destinação final e a coleta seletiva dos resíduos sólidos. Polêmica, 13(3), 1372-1383. https://www.e-publicacoes.uerj.br/index.php/polemica/article/view/11669/9146.

Bardin, L. (2016). Análise de Conteúdo. Almedina Brasil.

Brasil. (1988). Constituição da República Federativa do Brasil. http://www.planalto.gov.br/ccivil_03/constituicao/constituicao.htm.

Brasil. (2017). Ministério da Saúde. Lixo mal descartado pode causar acidentes e doenças. http://www.blog.saude.gov.br/index.php/53136-lixo-mal-descartadopode-causar-acidentes-e-doencas.

Borlina Tanaue, A. C., Mendes Bezerra, D., Cavalheiro, L., \& Pisano, L. C. (2015). Lixo eletrônico: agravos a saúde e ao meio ambiente. Ensaios e Ciência: Ciências Biológicas, Agrárias e da Saúde, 19(3), 130-134. https://www.redalyc.org/pdf/260/26042169006.pdf.

Cafure, V. A. \& Patriarcha-Graciolli, S. R. (2015). Os resíduos de serviço de saúde e seus impactos ambientais: uma revisão bibliográfica. Interações (Campo Grande), 16(2), 301-314. http://www.scielo.br/scielo.php?script=sci_arttext\&pid=S1518-70122015000200301\&lng=pt\&nrm=iso.

Capponi, N. F., Ahlert, A., Dal'sta, D., Fiirst, C. (2021). Educação ambiental e Agenda 2030: percepção de gestores de uma rede de ensino básico e superior privado. Research, Society and Development. 10(3), 1-15. https://rsdjournal.org/index.php/rsd/article/view/12895/11692.

Fröhlich, B. (2016). Impactos ambientais do descarte dos resíduos sólidos dos serviços de saúde. [Trabalho de Conclusão de Curso, Universidade Federal da Fronteira Sul]. https://rd.uffs.edu.br/handle/prefix/366. 
Research, Society and Development, v. 10, n. 3, e28610313206, 2021

(CC BY 4.0) | ISSN 2525-3409 | DOI: http://dx.doi.org/10.33448/rsd-v10i3.13206

Homrich, B. de S, Fernandes, C. R. P. \& Vieira J. R. G. O. (2014). O potencial tóxico do mercúrio e os impactos da gestão inadequada de seus resíduos ao meio ambiente e à saúde. Anais do V Congresso Brasileiro de Gestão Ambiental. http://www.ibeas.org.br/congresso/Trabalhos2014/III-094.pdf.

Masiero, M. (2020). Covid-19 e meio ambiente: descarte correto de resíduos. Hospital das Clínicas da Faculdade de Medicina de Botucatu. https://www.hcfmb.unesp.br/covid-19-e-meio-ambiente-descarte-correto-de-residuos/.

Oliveira, T.B \& Junior, A. C. G. (2016). Planejamento municipal na gestão dos resíduos sólidos urbanos e na organização da coleta seletiva. Eng Sanit Ambient. 21(1), 55-64. https://www.scielo.br/pdf/esa/v21n1/1413-4152-esa-21-01-00055.pdf.

Pereira, A. S., Shitsuka, D. M., Parreira, F. J., \& Shitsuka, R. (2018). Metodologia da pesquisa científica. UFSM. https://repositorio.ufsm.br/bitstream/ha ndle/1/15824/Lic_Computacao_Metodologia-Pesquisa-Cientifica.pdf?sequence=1.

Ramos, D. A. B. (2013). Impasses e dificuldades na gestão de resíduos de serviços de saúde: estudo de caso no município de Araçatuba - SP. [Dissertação de Mestrado, Escola Nacional de Saúde Pública Sérgio Arouca]. https://www.arca.fiocruz.br/bitstream/icict/35250/2/ve_Domicele_Aparecida_ENSP_2013.

Santos Junior, E. P., Batista, R. R. A. M., Almeida, A. T. F. \& Abreu, R. A. A. (2014). Acidente de trabalho com material perfurocortante envolvendo profissionais e estudantes da área da saúde em hospital de referência. Rev Bras Med Trab. 13(2), 69-75. http://www.anamt.org.br/site/upload_arquivos/rbmt_volume_13_n\%C2\%BA_2_29320161552145795186.pdf. 